\title{
Wind Turbine Rotor Simulation via CFD Based Actuator Disc Technique Compared to Detailed Measurement
}

\author{
Esmail Mahmoodi a*, Ali Jafari b, Alireza Keyhanib \\ aDepartment of Mechanical Engineering of Biosystems, University of Shahrood, Shahrood, Iran \\ ${ }^{b}$ Department of Mechanical Engineering of Biosystems, University of Tehran, Alborz, Iran
}

\begin{abstract}
In this paper, a generalized Actuator Disc (AD) is used to model the wind turbine rotor of the MEXICO experiment, a collaborative European wind turbine project. The AD model as a combination of CFD technique and User Defined Functions codes (UDF), so-called UDF/AD model is used to simulate loads and performance of the rotor in three different wind speed tests. Distributed force on the blade, thrust and power production of the rotor as important designing parameters of wind turbine rotors are focused to model. A developed Blade Element Momentum (BEM) theory as a code based numerical technique as well as a full rotor simulation both from the literature are included into the results to compare and discuss. The output of all techniques is compared to detailed measurements for validation, which led us to final conclusions.
\end{abstract}

Keywords: Wind Turbine, Rotor Performance, Actuator Disc Theory, BEM Theory, Full Rotor Simulation.

Article History: Received May 02, 2015; Received in revised form August 16, 2015; Accepted September 12, 2015; Available online How to Cite This Article: Mahmoodi, E., Jafari, A. and Keyhani, A. (2015). Wind Turbine Rotor Simulation via CFD Based Actuator Disc Technique Compared to Detailed Measurement. Int. Journal of Renewable Energy Development ,4(3), 205-210.

http://dx.doi.org/10.14710/ijred.4.3.205-210

\section{Introduction}

A lot of numerical and analytical techniques are developed to model wind turbine rotors: Blade Element Momentum (BEM) theory as the most popular method, full rotor or Direct Modeling (DM) as the soundest method to model the rotor and wake, and generalized Actuator Disc (AD), Actuator Line (AL) and Actuator Surface (AS) as the new developed models for wind turbine assessment. Inherently, the DM, AD, AL and AS methods have been based on Computational Fluid Dynamic (CFD) and mostly need a Navier-Stocks domain solver. The reader is referred to a review paper done by Sanderse et al. to get deep information in this regard (Sanderse et al. 2011).

Rotor prediction methods based on the actuator disc concept use the principle of representing rotors by equivalent forces distributed on a permeable disc of zero thickness in a flow domain (Mikkelsen 2003). The concept was introduced by Froude (Froude 1889) as a continuation of the work of Rankine (Rankine 1865) on momentum theory of propellers. Although the BEM method is the only method used routinely by industry, a large variety of advanced rotor predictive methods have been developed. Generally, the methods can be categorized into in-viscid models that demand the use of tabulated airfoil data, and viscous models based on either viscous in-viscid procedures or Navier-Stokes algorithms (Mikkelsen 2003).

The generalized actuator disc method represents a straightforward inviscid extension of the BEM technique. The main difference is that the annular independence of the BEM model is replaced by the solution of a full set of Euler or Navier-Stokes equations. Axisymmetric versions of the method have been developed and solved either by analytical/semianalytical methods (Wu 1962, Hough and Ordway 1965, Conway 1995, Conway 1998) or by finite difference / finite volume methods (Sørensen and Myken 1992, Sørensen and Kock 1995, Madsen 1996, Sørensen et al. 1998). The main goal of this study is the modeling of the loads and performance of the MEXICO rotor based on

${ }^{*}$ Corresponding author:

Email: esmahmoodi@shahroodut.ac.ir 
Citation: Mahmoodi, E., Jafari, A. and Keyhani, A. (2015). Wind Turbine Rotor Simulation via CFD Based Actuator Disc Technique Compared to Detailed Measurement. Int. Journal of Renewable Energy Development ,4(3), 205-210, http://dx.doi.org/10.14710/ijred.4.3.205-210

P a g e | 206

the combination of blade element momentum calculation and numerical solution. Solution of the axisymmetric version of the actuator disc via the finite volume method is employed in current research to feed momentums inside the disc domain and to get convergence on the rotor.

\section{MEXICO Experiment}

As a European supplement, the MEXICO (Model Experiments in Controlled Conditions) project (Snel et al. 2007, Schepers and Snel 2008) funded by the European Commission was formed (Shen et al. 2011). The rotor was tested in the DNW German-Dutch wind tunnel that called Large Low speed Facility (LLF) wind tunnel. The LLF wind tunnel is a closed return circuit between the collector and the compressor nozzle, and the MEXICO rotor is placed at a distance of $7 \mathrm{~m}$ from the edge of the nozzle (see Fig.1).

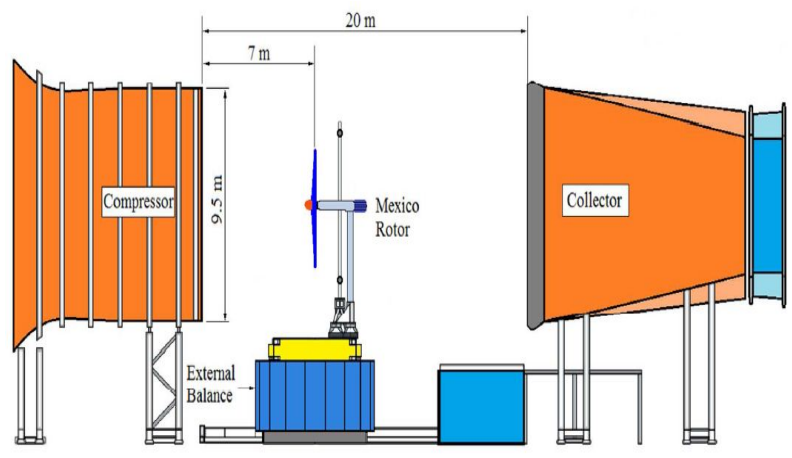

Fig. 1 Assembly of MEXICO rotor inside the LLF $9.5 \times 9.5 \mathrm{~m} 2$ open-jet test section.

The chosen measurements were performed with a tunnel wind speed of $10 \mathrm{~m} \mathrm{~s}-1$ (rotor turbulent state), $15 \mathrm{~m} \mathrm{~s}-1$ (rotor design state) and $24 \mathrm{~m} \mathrm{~s}-1$ (rotor blade stall state), corresponding to tip speed ratios of $\lambda=10.0$, $\lambda=6.7$ (the design tip speed ratio) and $\lambda=4.2$, respectively. Thrust and power productions are measured in a full range of wind speeds. For all measurements used in this work, the pitch angle is set at -2.3 degrees and rotor speed is set at $425.5 \mathrm{RPM}(\omega=$ 44.45 $\mathrm{rad} \mathrm{s}-1)$.

Each blade is composed of a cylinder, the inner $4.4 \%$ of the span; a DU91-W2-250 air foil, from $11.8 \%$ to $40 \%$ span; a RISOE-A1-21 air foil, from $50 \%$ to $62 \%$ span and a NACA $64-418$ air foil, from $72 \%$ to $100 \%$ span, with three transitional zones between the air foils. Fig.2 shows the air foil profiles used in the wind turbine blade.

The 2D aerodynamic coefficients $\left(C_{l}\right.$ and $C_{d}$ vs angle of attack) are required as an input of simulation models in this investigation. They were previously measured in the $2 \mathrm{D}$ wind tunnel tests.

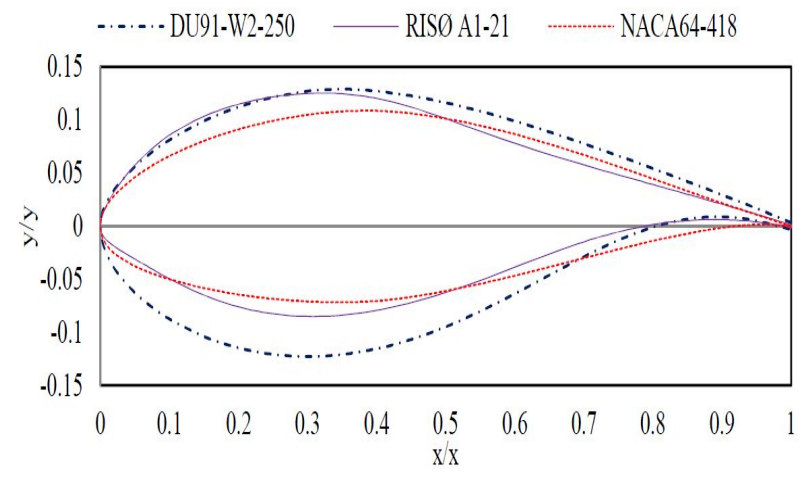

Fig. 2 Three Airfoil profiles used in Mexico rotor.

\section{Theoretical Aspect of the UDF/AD Model}

Equations for conservation of mass and momentum are solved in calculations. Conservation of momentum in the laminar form of the Navier-Stokes equations are introduced as LNS model.

The external body force of the LNS model contains model-dependent source terms such as porous-media or user-defined sources that are substituted by UDF source Macros in the actuator disc domain in this study. The Reynolds-Averaged Navier-Stokes (RANS model) equations govern the transport of the averaged flow quantities, with the whole range of the scales of turbulence being modeled. Flow quantities such as velocity and pressure are split in an average and a fluctuation, the so-called Reynolds decomposition. The Reynolds decomposition is substituted into the NavierStokes equations which are then averaged. Based on an analogy with laminar flow it states that the Reynolds stress tensor can be related to the mean velocity gradients via a turbulent eddy viscosity. Many different turbulent models have been used by researchers to calculate eddy viscosity. The standard $k-\varepsilon$ model is a two equation model often encountered in wind energy wake applications (Sanderse et al. 2011). Therefore a standard $k-\varepsilon$ turbulent model is used to compute eddy viscosity in the wake state of the RANS method in the current study.

An 0-Grid structured mesh is used to reach a reliable momentum distribution inside the Navier-Stokes domain of the actuator disc. The mesh of the disc is structured via 36 radial divisions from hub to tip, 72 azimuthal divisions, and 2 axial divisions. A hybrid mesh is used in the wind tunnel. Quad-dominant method is used to make surface grids and Tetra-mixed method is used for meshing volumes of the test section. Input and output channels are meshed in a structured way. Afterward, they are added to the test section to complete model of the wind tunnel. Finally, a cylinder carrying the disc is merged inside the wind tunnel to accomplish the final grid containing 6084344 cells and 1710361 nodes. 
The mathematical calculation of momentums acted as an external body force in the Navier-Stokes equations are the basic difference between this study and research of Rethore et al. (Rethore et al. 2010).

$$
\begin{aligned}
& f_{x}=-\rho V_{r e l}{ }^{2} B c\left(C_{l} \sin \varphi-C_{d} \cos \varphi\right)(4 \pi r t)^{-1} \sin \theta \\
& f_{y}=-\rho V_{r e l}{ }^{2} B c\left(C_{l} \sin \varphi-C_{d} \cos \varphi\right)(4 \pi r t)^{-1} \cos \theta \\
& f_{z}=-\rho V_{r e l}{ }^{2} B c\left(C_{l} \cos \varphi+C_{d} \sin \varphi\right)(4 \pi r t)^{-1} \\
& \varphi=\arctan \left(\frac{w}{V_{r e l}}\right), V_{r e l}=\sqrt{(r \omega+(v \cdot \cos \theta-w \cdot \sin \theta))^{2}+u^{2}}
\end{aligned}
$$

In equations $(1-4), \rho$ is air density, $V_{\text {rel }}$ is relative velocity of the local airfoil, $B$ is blade number, $c$ is the local chord, $C_{l}$ and $C_{d}$ are lift and drag coefficient of the airfoil, $\varphi$ is flow angle, $\theta$ is azimuthal angle of the element at the disc plan, $r$ is local radius of the element, $t$ is rotor thickness, $\omega$ is angular velocity of the rotor (44.45 rad s-1 ), $u, v$ and $w$ are axial (z-direction), vertical (x-direction) and horizontal (y-direction) velocity of the element center, respectively, $x$ and $y$ are vertical and horizontal distance from the element center to the rotor center of the disc plan. In these equations, lift and drag coefficient are calling for local 2D airfoil data. Velocity components of each element $(u$, $v, w)$ are measured from the Navier-Stokes domain in the iteration process by using UDF codes.

\section{Methodology}

The steps taken in order to develop an actuator disc model for the performance simulation of the wind turbine rotor are as follows:

- Geometry and aerodynamic specifications of the rotor are interpolated for being employable in continuing the recalling process during the iterations. In the transition areas, a linear function is finding the aerodynamic coefficients in the solution process.

- The User-Defined-Functions technique based on blade element analysis, including sub-functions of the geometry and aerodynamic specifications are coded to calculate external volume forces (the vector $f$ in equation (5) as follow) as momentum terms in the Navier-Stokes equations.

$$
\frac{\partial}{\partial t}(\rho \vec{u})+\nabla \cdot(\rho \vec{u} \vec{u})=-\nabla p+\nabla \cdot \mu\left(\left(\nabla \vec{u}+\nabla \vec{u}^{T}\right)-\frac{2}{3} \nabla \cdot \vec{u} I\right)+\vec{f}
$$

where $u$ is the flow vector, $p$ is the static pressure, $f$ vector is the external body forces (e.g., that arise from interaction with the dispersed phase). The term multiplied to $\mu$ is shear stress tensor, where, $\mu$ is the molecular viscosity, $I$ is the unit tensor, and the second phase on the right hand side of the term is the effect of volume dilation. The external body force, contains model-dependent source terms such as porous-media or userdefined sources that are substituted by UDF source Macros in the actuator disc domain in this study.

- $\quad$ The commercial CFD code Fluent (version 6.1) was used as flow solver, with the grid being created in ANSYS - ICEM CFD. The maximum value of Y-plus is under 10 percent in the whole grid. Refinement of the mesh is repeated since the independency of the grid is obtained.

- As the air flow can passes through the rotor, the boundary condition around the disc (interface between the disc the outer domain) is set as permeable wall. Boundary condition around the wind tunnel walls is set up with a shear less wall function.

- Both LNS and RANS approaches are set up in different up-coming wind speeds in the range of the experiment. The upcoming wind speed is used as the basis of the initial condition value in each solution. The wind speed should be reached at $7 \mathrm{~m}$ distance from the front of the disc in the upstream (output flow of the wind tunnel nozzle (see Fig.1)). So, with respect to the conservation of the mass flow and having the areas of the wind tunnel inlet and the nozzle, the initial condition is calculated for definition in each solution.

- Simple pressure-velocity coupling method described in reference (FLUENT 2006) are used to avoid pressure-velocity decoupling since external volume forces acting on the disc.

- Conservations of mass and momentum (equation (6) as follow) are used for the both LNS and RANS models as the criterions of the convergence process of solutions. For the RANS model, kinetic energy and dissipation ratio are also used to monitor the convergence process.

$\frac{\partial \rho}{\partial t}+\nabla \cdot(\rho \vec{u})=S_{m}$

where $S_{m}$ is external masses from another phases of the flow. So, the $S_{m}$ term as well as the density term $(\partial \rho / \partial t)$, both are zero because of incompressible single phase flow assumption in wind energy story.

- Equations (1), (2) and (3) are applied along the axial, horizontal and vertical directions, respectively, on the external forces of the NavierStokes equations (equation (5)). Use of these equations in the grid solution distinguishes the disc domain from the outer domain.

- After the solutions are converged, the results are compared to a BEM technique and a full rotor model from the literatures then they are discussed and validated against experimental data. 
Citation: Mahmoodi, E., Jafari, A. and Keyhani, A. (2015). Wind Turbine Rotor Simulation via CFD Based Actuator Disc Technique Compared to Detailed Measurement. Int. Journal of Renewable Energy Development ,4(3), 205-210, http://dx.doi.org/10.14710/ijred.4.3.205-210

P a g e | 208

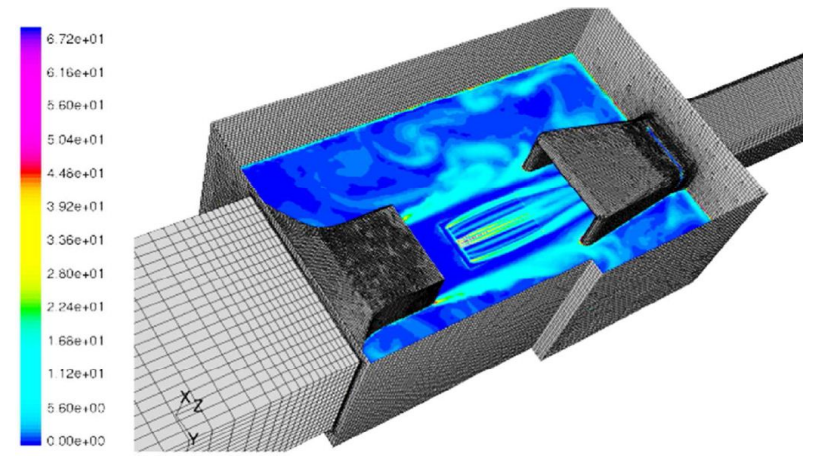

Fig. 3 Vorticity magnitude at $15 \mathrm{~m} \mathrm{~s}-1$ of wind speed.
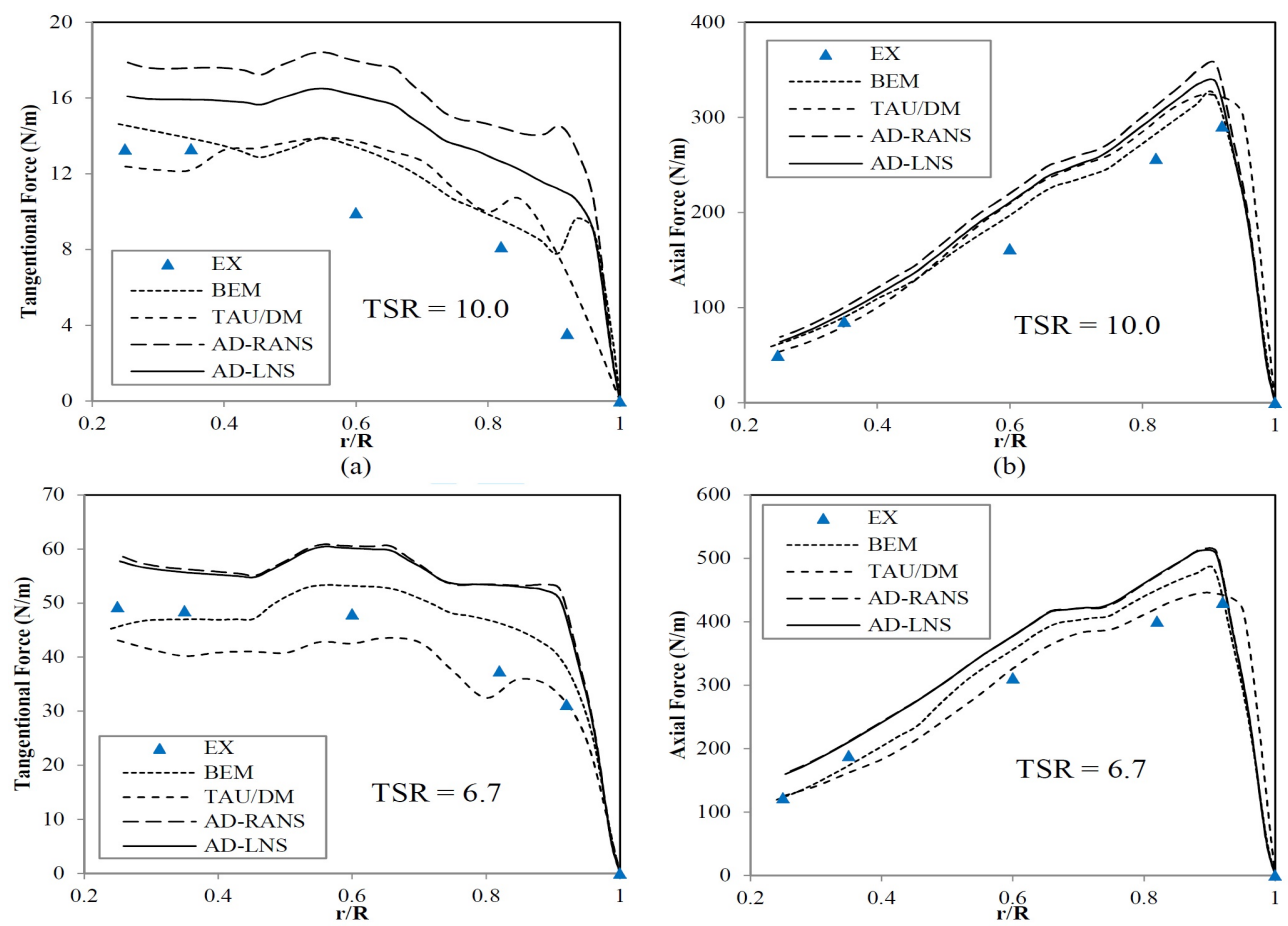

(c)

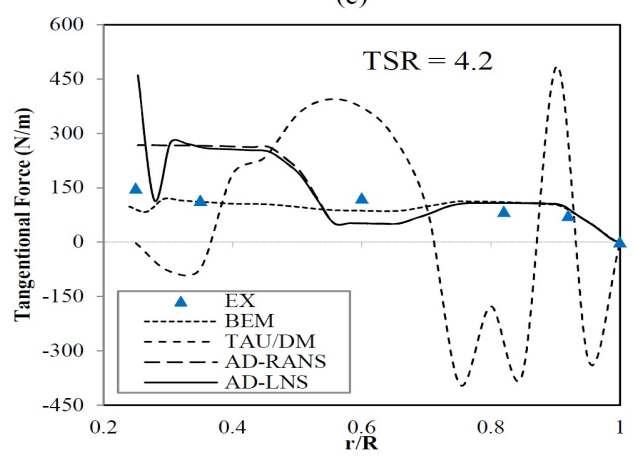

(e)

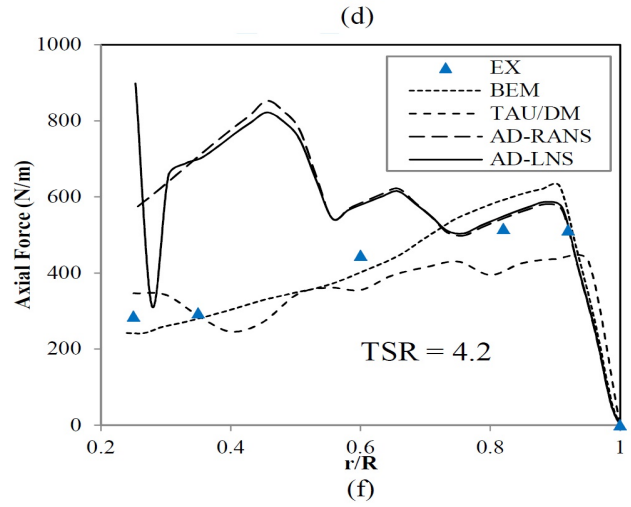

Fig. 4 Comparison of blade loads of UDF/AD models with TAU/DM, BEM and experimental data: (a)\&(b) in TSR=10.0, (c)\&(d) in TSR=6.7 and (e)\&(f) in TSR=4.2. 


\section{Results and Discussion}

Below, selected results from the simulations are presented and compared with measurements. First, a vorticity distribution inside the DNW/LLF wind tunnel is presented. Afterwards, the corresponding momentum force along the blade and rotor performance are compared and discussed.

High-speed turbulence and vortices can be difficult to see directly. Distribution of vorticity magnitude inside the wind tunnel, since the actuator disc is placed instead of the rotor, is plotted in Fig.3. As seen, the edge of the compressor nozzle, the rotor's tip and root are the main sources of shear layers. Inside the test section, large scale vortices are released the edge of the compressor nozzle. As seen, the rotor jet is surrounded by its shear layer. This shows the rotor jet can be affected by the test section, although it's an open jet. The reader who are interested in the wake is referred to (Mahmoodi et al. 2015) where it is simulated and discussed against the full Paritcle Image Velocimetry measurement of the MEXICO experiment.

Both laminar and turbulent simulations of UDF/AD model result in almost the same values for axial and tangential forces (see Fig.4). Both AD and DM exceed the experimental measured values, especially when $\mathrm{r} / \mathrm{R}$ $>0.6$. Jump calculated in the middle of the blade can be originated of wrong airfoil data measurement, as Shen et al. also discussed in actuator line simulation of the MEXICO experiment (Shen et al. 2011). The difference between $\mathrm{AD}$ and DM-Model is more pronounced for the tangential forces. Accuracy of all models is increasing at the design wind speed (TSR=6.7). As wind speed increases up to $24 \mathrm{~m} \mathrm{~s}-1$ (TSR $=4.2$ ), the result of all CFD models exhibits more instability compare to BEM calculation. Using RANS turbulent model inside the AD did not improve the results, although it's consuming more time and cost. This indicates that RANS model is not proper for simulating volume forces in 3D domain.

Fig.5 shows performance parameters of the rotor. Because of weakness of the AD-RANS model discussed above, it's excluded from modeling the performance. As seen, BEM calculation and AD-LNS model exhibit significant prediction of power production (Fig.5-a). Around the design state, (TSR=6.7) ability of the ADLNS model is more pronounced comparing to the BEM model. Considering the whole geometry of the wind tunnel in the AD-LNS can be the reason of this prominence. The full rotor model exhibits a big minus offset error in the calculation of the power production calculation.

As seen in Fig.5, both power (Cp) and thrust (Ct) coefficients are well predicted. It's obviously can be seen that $\mathrm{Cp}$ is precisely estimated by LNS model around the designing state of the wind turbine (where $\mathrm{Cp}$ is maximized, TSR $\cong 7$ ). In the turbulent state (high TSR) and stall state (low TSR) the BEM model is also exhibits its ability to predict the output power. Thrust coefficient can be divided into two areas: before and after designation TSR, (A) and (B) areas respectively (please look at Fig.5-b). In area $A$, where the rotor is under higher wind speeds (low TSR), TAU/DM and BEM technique behave the same and with a plus gain error. The AD-LNS model exhibits a plus offset error here. In area $B$, where rotor is under lower wind speeds (high TSR), the AD-LNS and BEM model behave the same and with a combination of plus gain and offset errors. Where the TAU/DM model continues with a plus gain error.

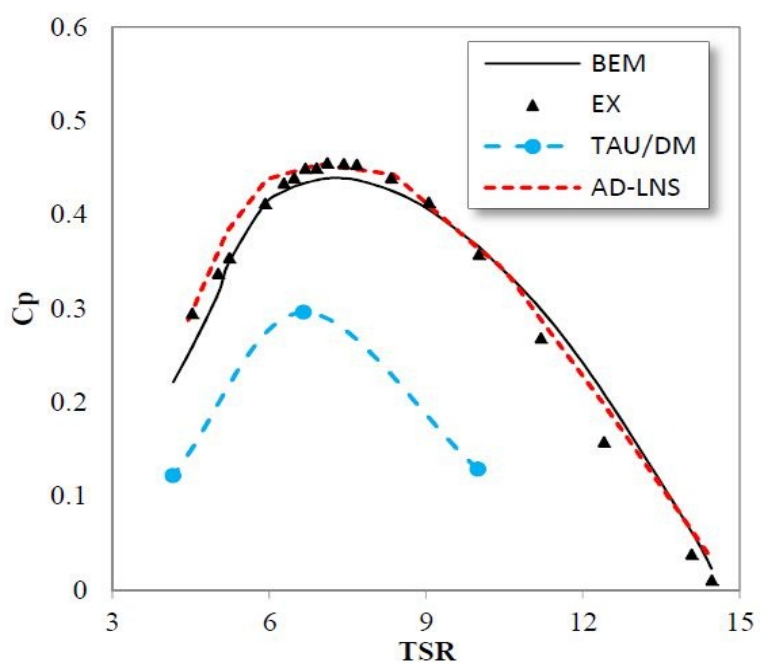

(a)

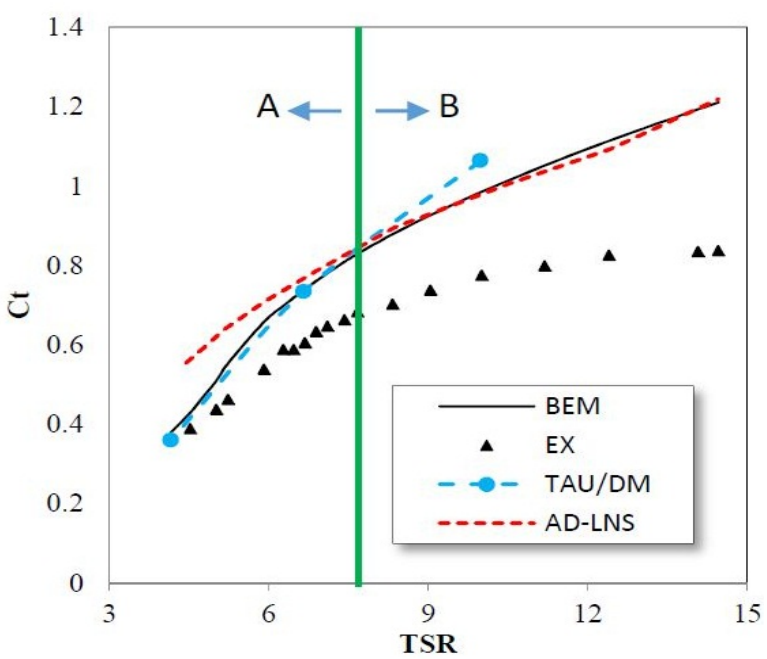

(b)

Fig. 5 Performance coefficients of the rotor: (a) power coefficient, (b) thrust coefficient.

As indicated in Fig.6 for thrust prediction, the ADLNS technique shows plus offset error about 0.147 unit of Ct. Both BEM and TAU/DM models exhibit plus gain error about 0.31 unit of slope, since the slope is more pronounced for the TAU/DM model in higher Ct. These 
indicate that studying on correction methods for predicting thrust must be focused on offset errors in actuator disk models and gain errors for both BEM and full rotor techniques.

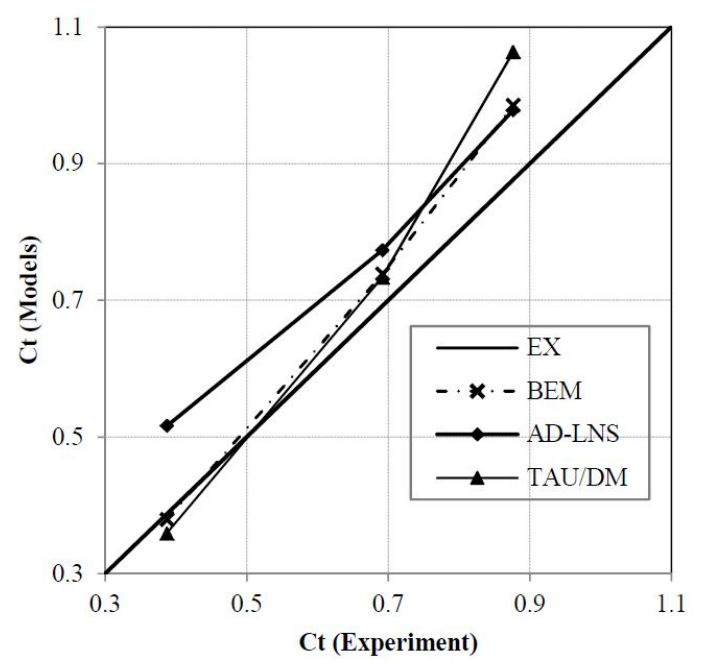

Fig. 6 Thrust coefficients of theoretical models against measurement data.

\section{Conclusion}

The AD-LNS and AD-RANS models studied in this work were validated against the detailed measurements performed during the European MEXICO project. A BEM model and a full rotor calculation of the MEXICO project from the literature were included in the diagrams to compare and discuss in detail. Following are the main conclusions of this study:

- Visualization of the vorticity inside the open jet test section exhibited that the shear layer released from the edge of the compressor nozzle surrounded the rotor jet. This could be one of the main sources of errors of the experiment, especially while measuring wake via PIV instruments.

- In general, good agreements between the AD computations and the MEXICO experiments are seen for both the loading on the blade and the performance of the rotor.

- We found that all computations discussed above are good at the design state. Since for higher wind speeds, a developed BEM model predicts better than CFD models.

- Both BEM and AD-LNS models exhibit significant prediction of power production. In the design state, the ability of the AD-LNS as a CFD model, shows better results than BEM as a code-based technique.

- For simulating the rotor drag, it seems study on corrections must be focused on offset errors in actuator disk models and gain errors for both BEM and full rotor techniques.

\section{Acknowledgments}

We are very thanking full of University of Tehran, University of Shahrood and University of Applied Science, Kiel for funding this research and giving us scientific consultation.

\section{References}

Conway, J.T. (1995) Analytical solutions for the actuator disc with variable radial distribution of load. Journal of Fluid Mechanics, 297: 327-355

Conway, J.T. (1998) Prediction of the performance of heavily loaded propellers with slipstream contraction. CASI J., 44: 169-174.

Fluent (2006) Theory guide: Pressure-velocity coupling. ANSYS Documentations, ANSYS.

Froude, R.E. (1889) On the part played in propulsion by differences of fluid pressure. Trans. Inst. Naval Architects, 30: 390.

Hough, G. \& Ordway, D. (1965) The generalized actuator disk. Developments in theoretical and applied mechanics, 2: 317-336.

Madsen, H.A. (1996) CFD analysis of the actuator disc flow compared with momentum results. 10th IEA Meeting on Aerodynamics. University of Edinburgh.

Mahmoodi, E., Jafari, A. \& Keyhani, A. (2015) Near wake modeling of a wind turbine particle image velocimetry experiment Iranica Journal of Energy and Environment, 6(3), 195-206.

Mikkelsen, R. (2003) Actuator disc methods applied to wind turbines. PhD Thesis, Technical University of Denmark.

Rankine, W.J.M. (1865) On the mechanical principles of the action of propellers. Trans. Inst. Naval Architects, 6: 13-39.

Rethore, P.E., Sørensen, N.N. \& Madsen, H.A. (2010) Modelling the mexico wind tunnel with cfd. IEA wind, MexNext meeting. Forth, Heraklion, Crete, Greece.

Sanderse, B., Van Der Pijl, S.P.V. \& Koren, B. (2011) Review of CFD for wind-turbine wake aerodynamics. Wind Energy, 14 (7), 799-819.

Schepers, J.G. \& Snel, H. (2008) Model experiments in controlled conditions. ECN Project Report

Shen, W.Z., Zhu, W.J. \& Sørensen, J.N. (2011) Actuator line/navierstokes computations for the mexico rotor: Comparison with detailed measurements. Wind Energy, 15(5), 811-825.

Snel, H., Schepers, J.G. \& Montgomerie, B. (2007) The mexico (model experiments in controlled conditions): The database and first results of data process and interpretation. Journal of Physics: Conference Series, 75, 012014.

Sørensen, J.N. \& Kock, C.W. (1995) A model for unsteady rotor aerodynamics. Journal of Wind Engineering and Industrial Aerodynamics, 58: 259-275.

Sørensen, J.N. \& Myken, A. (1992) Unsteady actuator disc model for horizontal axis wind turbines. Journal of Wind Engineering and Industrial Aerodynamics, 39: 139-149.

Sørensen, J.N., Shen, W.Z. \& Munduate, X. (1998) Analysis of wake states by a full-field actuator disc model. Wind Energy, 1 (2), 7388.

$\mathrm{Wu}$, T. (1962) Flow through a heavily loaded actuator disc. Schiffstechnik, 9 (47), 134-138. 